\section{Production and detection of cold antihydrogen atoms}

\author{
M. Amoretti ${ }^{\star}$, C. Amsler $\dagger$, G. Bonomi $\ddagger \varsigma$, A. Bouchta $\ddagger$, P. Bowe $\|$, \\ C. Carraro*, C. L. Cesarq, M. Charlton\#, M. J. T. Collier\#, M. Doserł, \\ V. Filippini $\_$, K. S. Fine $\ddagger$, A. Fontana ${ }^{* *}$, M. C. Fujiwara $\dagger \dagger$, \\ R. Funakoshi $\dagger \dagger$, P. Genova $\imath^{* *}$, J. S. Hangst $\|$, R. S. Hayano $\dagger \dagger$, \\ M. H. Holzscheiter $\neq$, L. V. Jørgensen\#, V. Lagomarsino $\neq_{\ddagger \neq}$, R. Landua $\neq$,

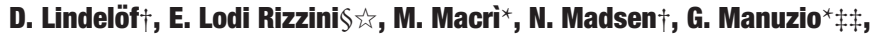 \\ M. Marchesotti $\_$, P. Montagna ${ }^{*}{ }^{*}$, H. Pruys $\dagger$, C. Regenfus $\dagger$, P. Riedler $\neq$, \\ J. Rochet $\dagger \#$, A. Rotondi $\tau^{* *}$, G. Rouleau $\neq \#$, G. Testera ${ }^{\star}$, A. Variola ${ }^{\star}$, \\ T. L. Watson\# \& D. P. van der Werf\#
}

${ }^{*}$ Istituto Nazionale di Fisica Nucleare, Sezione di Genova, and di Fisica, Università di Genova, 16146 Genova, Italy

$\dagger$ Physik-Institut, Zürich University, CH-8057 Zürich, Switzerland

$\ddagger E P$ Division, CERN, CH-1211 Geneva 23, Switzerland

$\$$ Dipartimento di Chimica e Fisica per l'Ingegneria e per i Materiali, Università di Brescia, 25123 Brescia, Italy

II Department of Physics and Astronomy, University of Aarhus, DK-8000 Aarhus C, Denmark

II Instituto de Fisica, Universidade Federal do Rio de Janeiro, Rio de Janeiro 21945-970, and Centro Federal de Educação Tecnologica do Ceara, Fortaleza 60040-531, Brazil

\# Department of Physics, University of Wales Swansea, Swansea SA2 8PP, UK is Istituto Nazionale di Fisica Nucleare, Sezione di Pavia, and ${ }^{* \star}$ Dipartimento di Fisica Nucleare e Teorica, Università di Pavia, 27100 Pavia, Italy

$\dagger \dagger$ Department of Physics, University of Tokyo, Tokyo 113-0033, Japan

A theoretical underpinning of the standard model of fundamental particles and interactions is CPT invariance, which requires that the laws of physics be invariant under the combined discrete operations of charge conjugation, parity and time reversal. Antimatter, the existence of which was predicted by Dirac, can be used to test the CPT theorem-experimental investigations involving comparisons of particles with antiparticles are numerous $^{1}$. Cold atoms and anti-atoms, such as hydrogen and antihydrogen, could form the basis of a new precise test, as CPT invariance implies that they must have the same spectrum. Observations of antihydrogen in small quantities and at high energies have been reported at the European Organization for Nuclear Research $(\mathrm{CERN})^{2}$ and at Fermilab ${ }^{3}$, but these experiments were not suited to precision comparison measurements. Here we demonstrate the production of antihydrogen atoms at very low energy by mixing trapped antiprotons and positrons in a cryogenic environment. The neutral anti-atoms have been detected directly when they escape the trap and annihilate, producing a characteristic signature in an imaging particle detector.

The current experiment, called ATHENA, seeks to compare the frequency of the 1S-2S electronic transition (ground state to first excited state) in hydrogen with that in antihydrogen. This frequency has been measured in hydrogen ${ }^{4}$ to an accuracy of 1.8 parts in $10^{14}$. Obtaining similar precision in antihydrogen is possible in principle, but only if very cold (of the order of a few kelvin) anti-atoms are available.

Located adjacent to the antiproton decelerator ${ }^{5}(\mathrm{AD})$ ring at the CERN laboratory in Geneva, the ATHENA apparatus comprises four main subsystems: the antiproton catching trap, the positron accumulator, the antiproton/positron mixing trap, and the antihy- drogen annihilation detector. All traps in the experiment are variations on the Penning trap ${ }^{6}$, which uses an axial magnetic field to transversely confine the charged particles, and a series of hollow cylindrical electrodes to trap them axially (Fig. 1a). The catching and mixing traps are adjacent to each other, and coaxial with a $3 \mathrm{~T}$ magnetic field from a superconducting solenoid. The positron accumulator has its own magnetic system, also a solenoid, of $0.14 \mathrm{~T}$. A separate cryogenic heat exchanger in the bore of the superconducting magnet cools the catching and mixing traps to about $15 \mathrm{~K}$. The ATHENA apparatus ${ }^{7}$ features an open, modular design that allows great experimental flexibility, particularly in introducing large numbers of positrons into the apparatus-an essential factor in the current work.

The catching trap ${ }^{8}$ slows, traps, cools and accumulates antiprotons. To cool antiprotons, the catching trap is first loaded with $3 \times 10^{8}$ electrons, which cool by synchrotron radiation in the $3 \mathrm{~T}$ magnetic field. Typically, the AD delivers $2 \times 10^{7}$ antiprotons having kinetic energy $5.3 \mathrm{MeV}$ and a pulse duration of $200 \mathrm{~ns}$ to the experiment at 100-s intervals. The antiprotons are slowed in a thin foil and trapped using a pulsed electric field. The antiprotons lose energy and equilibrate with the cold electrons by Coulomb interaction. The electrons are ejected before mixing the antiprotons with positrons. Each $\mathrm{AD}$ shot results in about $3 \times 10^{3}$ cold antiprotons for interaction experiments.
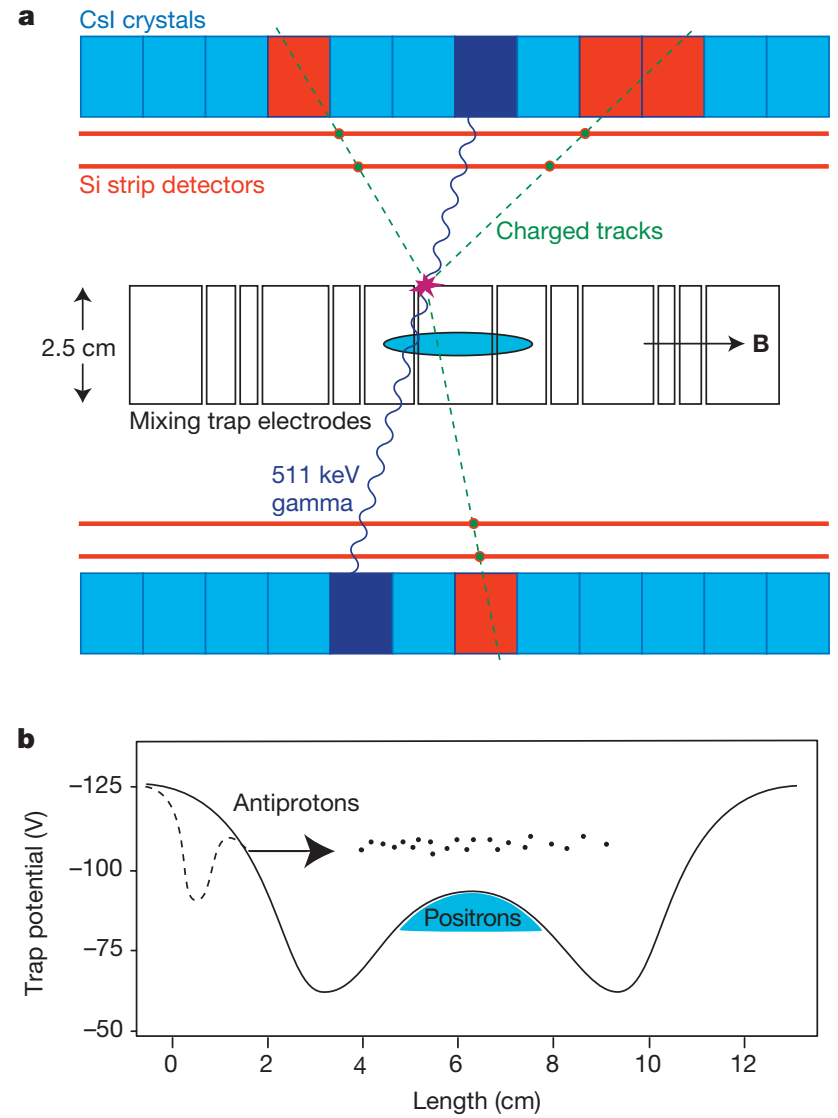

Figure 1 Central part of the ATHENA apparatus and trapping potential. a, Schematic diagram, in axial section, of the ATHENA mixing trap and antihydrogen detector. The cylindrical electrodes and the position of the positron cloud (blue ellipse) are shown. A typical antihydrogen annihilation into three charged pions and two back-to-back 511-keV photons is also shown. The arrow indicates the direction of the magnetic field. The detector active volume is $16 \mathrm{~cm}$ long and has inner and outer diameters of $7.5 \mathrm{~cm}$ and $14 \mathrm{~cm}$, respectively. $\mathbf{b}$, The trapping potential is plotted against length along the trap. The dashed line is the potential immediately before antiproton transfer. The solid line is the potential during mixing. 
The positron accumulator, based on a design detailed in ref. 9, slows, traps and accumulates positrons emitted from a radioactive source $\left(1.4 \times 10^{9} \mathrm{~Bq}^{22} \mathrm{Na}\right)$. Accumulation for $300 \mathrm{~s}$ yields $1.5 \times 10^{8}$ positrons ${ }^{10}, 50 \%$ of which are successfully transferred to the mixing trap where they cool by synchrotron radiation. The mixing trap has the axial potential configuration of a nested Penning trap ${ }^{11}$ (Fig. 1b), which permits two plasmas of opposite charge to come into contact. In ATHENA, the spheroidal cloud of positrons can be characterized by exciting and detecting axial plasma oscillations, as demonstrated by ref. 12 . Typical conditions are: $7 \times 10^{7}$ stored positrons, a radius of $2-2.5 \mathrm{~mm}$, a length of $32 \mathrm{~mm}$, and a maximum density of $2.5 \times 10^{8} \mathrm{~cm}^{-3}$.

Central to the observations reported here is the antihydrogen annihilation detector ${ }^{13}$ (Fig. 1a), situated coaxially with the mixing region, between the outer radius of the trap and the magnet bore. The detector is designed to provide unambiguous evidence for antihydrogen production by detecting the temporally and spatially coincident annihilations of the antiproton and positron when a neutral antihydrogen atom escapes the electromagnetic trap and strikes the trap electrodes. An antiproton typically annihilates into a few charged or neutral pions ${ }^{14}$. The charged pions are detected by two layers of double-sided, position-sensitive silicon microstrips. The path of a charged particle passing through both microstrip
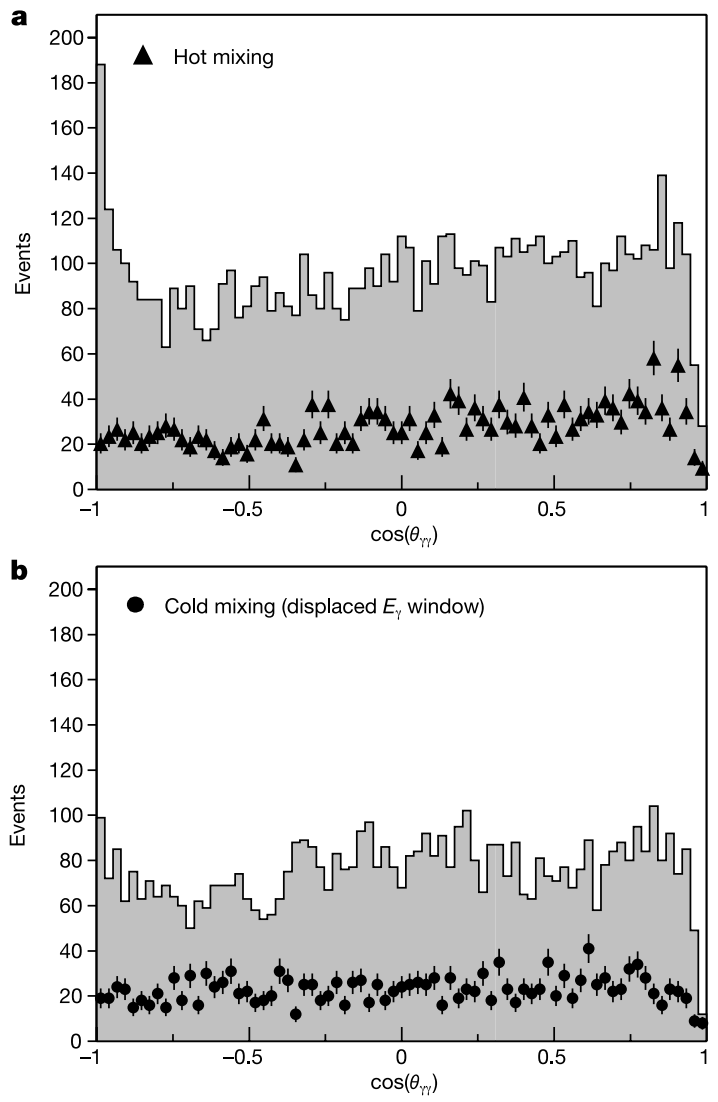

Figure 2 Experimental data. a, The number of events passing the selection criteria is plotted against the cosine of the opening angle $\theta_{\gamma \gamma}$ (see text for definition). The histogram is for cold mixing data (grey background). A total of 7,125 events, out of a sample of 103,270 reconstructed vertices, have two clean (but not necessarily back-to-back), detected photons in the 511-keV energy window. The data represent 165 mixing cycles. Filled triangles represent hot mixing data and are scaled by 1.6 to depict the same number of mixing cycles. b. The opening angle distribution (grey histogram) for antiproton-only data (99,610 vertices reconstructed, 5,658 clean events plotted). The filled circles represent cold mixing data, analysed using an energy $\left(E_{\gamma}\right)$ window displaced upward so as not to include the $511-\mathrm{keV}$ photo peak; no angular correlation of photons is seen. layers can be reconstructed, and two or more intersecting tracks allow determination of the position, or vertex, of the antiproton annihilation. The uncertainty in vertex determination is approximately $4 \mathrm{~mm}(1 \sigma)$ and is dominated by the unmeasured curvature of the charged pions' trajectories in the magnetic field. The temporal coincidence window is approximately $5 \mu \mathrm{s}$. The solid angle coverage of the interaction region is about $80 \%$ of $4 \pi$.

A positron annihilating with an electron yields two or three photons. The positron detector, comprising 16 rows, each row containing 12 scintillating, pure CsI crystals ${ }^{15}$, is designed to detect the two-photon events, consisting of two $511-\mathrm{keV}$ photons that are always emitted back-to-back. The energy resolution of the detector is $18 \%$ full-width at half-maximum (FWHM) at $511 \mathrm{keV}$, and the photo-peak detection efficiency for single photons is about $20 \%$.

The maximum readout rate of the whole detector is about $40 \mathrm{~Hz}$. Ancillary detectors include large scintillator paddles external to the magnet, and a thin, position-sensitive silicon diode through which the incident antiproton beam passes before entering the catching trap.

To produce antihydrogen atoms, we fill a positron well in the mixing region with about $7 \times 10^{7}$ positrons and allow them to cool to the ambient temperature $(15 \mathrm{~K})$. The nested trap is then formed around the positron well. Next, approximately $10^{4}$ antiprotons (three $\mathrm{AD}$ shots) are launched into the mixing region by pulsing the trap from one potential configuration (dashed line in Fig. 1b) to another (solid line). The mixing time is $190 \mathrm{~s}$, after which all particles are dumped and the process repeated. Events triggering the imaging silicon detector (three sides hit in the outer layer) initiate readout of both the silicon and the CsI modules. We refer to this procedure as 'cold mixing'.

A second type of procedure, 'hot mixing', involves radiofrequency heating of the axial motion of the positron plasma. During the mixing cycle with antiprotons, the positrons are maintained at a temperature of several thousand kelvin. The positron temperature is monitored using the plasma mode analysis technique of ref. 12. The two mechanisms of antihydrogen formation expected to be important in ATHENA, radiative recombination and three-body recombination ${ }^{16}$, should be effectively suppressed at the hot mixing temperature, although the positrons and antiprotons still interact.

For comparison and for investigating background processes, we loaded antiprotons into the interaction region without positrons and accumulated detector data. The resulting data set is referred to as 'antiproton only'.

For cold mixing, interaction of the two particle species is immediately apparent, as the kinetic energy of the antiprotons is rapidly reduced ( $<1 \mathrm{~s}$, an upper limit set by our current experimental method) to the level corresponding to the positron well. The cooling process is observed by lowering the wall of the antiproton well to various levels and recording the antiproton loss on the external scintillators. Such cooling has been reported previously ${ }^{17}$, but with a factor of 300 fewer positrons. Our measurements of antiprotons without positrons show no such cooling effect, also indicating effective removal of electrons.

At the start of cold mixing, we observe a rapid jump in the rate of antiproton annihilation, rising to a value that is tenfold higher than that for hot mixing. The positron loss during hot mixing is indistinguishable from that during cold mixing.

Subsequent observations are based on analysis of the annihilation detector data. Antiproton annihilation vertices are reconstructed from the silicon detector data using standard techniques ${ }^{18}$. For each vertex, we search for 'clean' evidence of 511-keV photons in the crystal data. A charged particle hit in a crystal, or an outer-layer silicon hit lying in the footprint of a crystal, excludes that particular crystal and its eight nearest neighbours. Next, we demand that exactly two of the remaining crystals have hits in an energy window around $511 \mathrm{keV}$, and that there are no hits of any energy adjacent to 


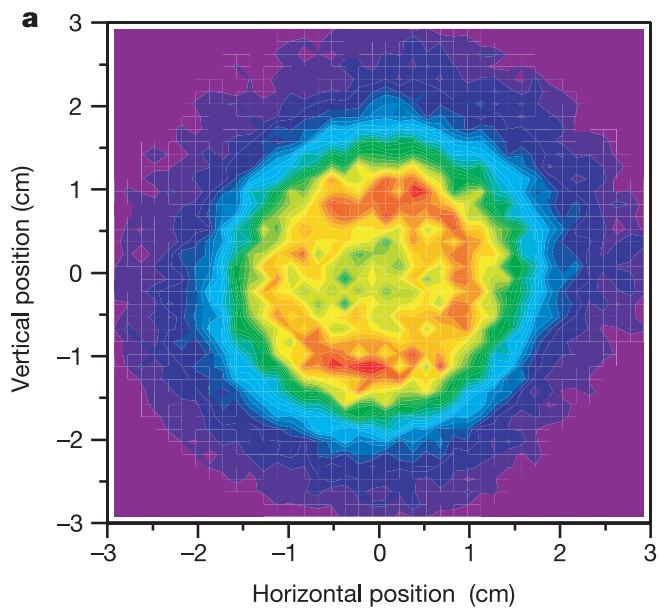

Figure 3 Colour contour plots of the distribution (obtained by projecting into the plane perpendicular to the magnetic field) of the vertex positions of reconstructed events. a, Cold mixing. All reconstructed antiproton annihilation vertices from the mixing region are plotted-no crystal cuts are applied. The trap inner radius is $1.25 \mathrm{~cm}$. The

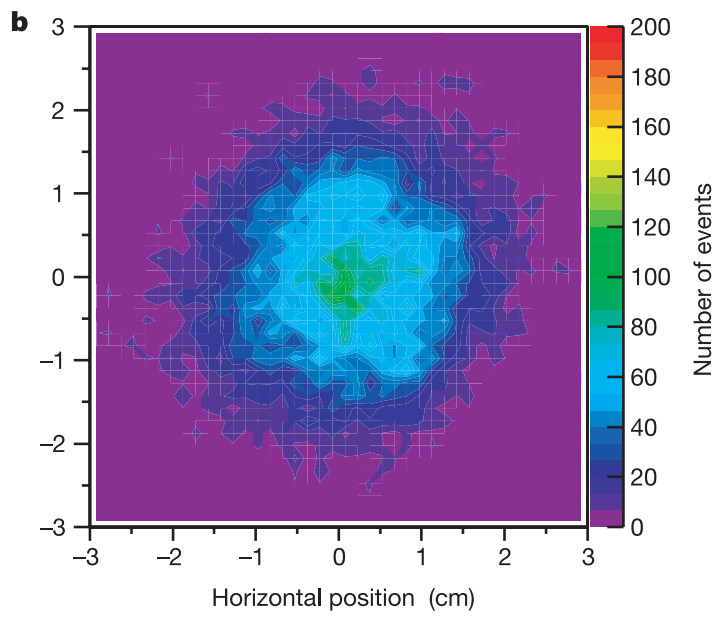

annihilations are centred on a slightly smaller radius, in agreement with our Monte Carlo simulations. (Some events appear to be outside of the trap radius owing to vertex reconstruction errors.) b. The same plot as above, but for hot mixing. These data are normalized to represent the same number of mixing cycles (165) as those in $\mathbf{a}$. these two crystals. Energy calibration data, measured for each individual crystal, are used in this test.

To search for antihydrogen in the sample of events having a vertex and two 'clean' photons, we consider the opening angle, $\theta_{\gamma \gamma}$, between the lines connecting the vertex point to the geometric centres of the two hit crystals. For an antihydrogen event, this angle should be $180^{\circ}$ (or $\cos \left(\theta_{\gamma \gamma}\right)=-1$ ). The opening angle distribution for reconstructed events detected during cold mixing is shown in Fig. 2a. The equivalent plots for hot mixing (Fig. 2a) and antiproton-only (Fig. 2b) data are also shown. Compared with the hot mixing and antiproton-only data samples, the cold mixing data display a marked enhancement of events close to $\cos \left(\theta_{\gamma \gamma}\right)=-1$, indicating antihydrogen production. Analysis of the cold mixing data using an energy window that does not include the 511-keV peak in the crystal spectra yields no evidence for antihydrogen (Fig. 2b).

The shape of the $\cos \left(\theta_{\gamma \gamma}\right)$ distribution predicted by Monte Carlo analysis of our detector for pure antihydrogen annihilation agrees well with the cold mixing measurement. In particular, the width of the peak close to $\cos \left(\theta_{\gamma \gamma}\right)=-1$ is the same for our data and for the Monte Carlo simulation. The dominant background for the experiment is caused by antiproton annihilations that contain a neutral pion, the decay of which produces electromagnetic showers in the material surrounding the trap and can lead to $511-\mathrm{keV}$ signals in the CsI crystals. Our antiproton-only data (Fig. 2b) rule out this process as a source of antihydrogen-like signal in our cold mixing data. The shape of a Monte Carlo simulation of antiproton-only events also agrees well with our measurement. Other sources of background, such as cosmic rays and background from the accelerator complex (AD injection and extraction) have also been excluded. The probability for accidental temporal coincidence of antiproton and positron annihilation is negligible during mixing, as determined from measured trigger rates for each part of the detector. Furthermore, Monte Carlo simulations of this background indicate no enhancement in opening angle at $180^{\circ}$. Correlated loss due to scattering of antiprotons on positrons outside of the spheroidal cloud is ruled out by rate and dynamical considerations.

Further confirmation of antihydrogen production can be seen in the cold mixing data if the distribution of all antiproton annihilation vertex positions is projected onto a plane perpendicular to the magnetic field (Fig. 3a). A clear image of the trap electrodes is obtained, consistent with neutral anti-atoms annihilating on the wall. This observation also implies that many more antihydrogen atoms are produced than are actually detected through their angularly correlated photons, a fact consistent with our Monte Carlo analysis. The vertex distribution for mixing with heated positrons has a very different shape and no evidence of annihilation on the trap wall (Fig. 3b). Rather, the antiprotons annihilate in the central region of the trap, probably owing to collisions with the residual gas or with trapped ions in the positron plasma. The average annihilation rate for hot mixing is about a factor of 4 smaller than that for cold mixing, whereas the peak ratio is about a factor of 10 smaller, as observed above.

If we consider cold mixing data only in the range $\cos \left(\theta_{\gamma \gamma}\right)<-0.95$, we have detected $131 \pm 22$ events with a reconstructed vertex and a pair of back-to-back, $511-\mathrm{keV}$ photons above a conservatively scaled antiproton-only background. With an upper limit of the detection and reconstruction efficiency of $2.5 \times 10^{-3}$, on the basis of Monte Carlo analysis, we estimate that at least 50,000 antihydrogen atoms were created during cold mixing. This can be compared with the total number of antiprotons mixed, about $1.5 \times 10^{6}$.

It is premature to discuss absolute production rates in our experiment, but it is noteworthy that the time distribution of the $\cos \left(\theta_{\gamma \gamma}\right)<-0.95$ events parallels the total annihilation rate during mixing. Further study is also needed to identify the mechanism of antihydrogen formation. We note that the present detection method does not identify the quantum state of the produced antihydrogen atoms. This is a question of considerable practical importance for future experiments involving trapping of neutral anti-atoms, as the demonstrated trapping techniques for hydrogen are only applicable to ground-state atoms.

Received 27 August; accepted 3 September 2002; doi:10.1038/nature01096. Published online 18 September 2002.

1. Hagiwara, K. et al. The review of particle physics. Phys. Rev. D 66, 010001 (2002).

2. Baur, G. et al. Production of antihydrogen. Phys. Lett. B 368, 251-258 (1996).

. Blanford, G. et al. Observation of atomic antihydrogen. Phys. Rev. Lett. 80, 3037-3040 (1998).

4. Niering, M. et al. Measurement of the hydrogen $1 \mathrm{~S}-2 \mathrm{~S}$ transition frequency by phase coherent comparison with a microwave cesium fountain clock. Phys. Rev. Lett. 84, 5496-5499 (2000).

5. Hellemans, A. Through the looking glass. Nature 406, 556-558 (2000).

6. Dehmelt, H. Experiments with an isolated subatomic particle at rest. Rev. Mod. Phys. 62, 525-530 (1990).

7. Fujiwara, M. C. et al. Producing slow antihydrogen for a test of CPT symmetry with ATHENA. Hyperfine Interactions (in the press).

8. Testera, G. et al. Toward the production of antihydrogen at rest in ATHENA. Nucl. Instrum. Methods A 461, 253-255 (2001). 
9. Surko, C. M., Gilbert, S. J. \& Greaves, R. G. Non-Neutral Plasma Physics (eds Bollinger, J. J., Spencer, R. L. \& Davidson, R. C.) Vol. 3, 3-12 (American Institute of Physics, New York, 1999).

10. Jørgensen, L. V., van der Werf, D. P., Watson, T. L., Charlton, M. \& Collier, M. J. T. Nonneutral Plasma Physics (eds Anderegg, F., Schweikhard, L. \& Driscoll, C. F.) Vol. 4, 35-44 (American Institute of Physics, New York, 2002).

11. Gabrielse, G., Rolston, S., Haarsma, L. \& Kells, W. Antihydrogen production using trapped plasmas. Phys. Lett. A 129, 38-42 (1988).

12. Tinkle, M. D., Greaves, R. G., Surko, C. M., Spencer, R. L. \& Mason, G. W. Low-order modes as diagnostics of spheroidal non-neutral plasmas. Phys. Rev. Lett. 72, 352-355 (1994).

13. Regenfus, C. A cryogenic silicon micro strip and pure-CsI detector for detection of antihydrogen annihilations. Nucl. Instrum. Methods A (in the press).

14. Bendiscioli, G. \& Kharzeev, D. Antinucleon-nucleon and antinucleon-nucleus interaction, a review of experimental data. Rivista Nuovo. Cim. 17(6), 1-42 (1994).

15. Amsler, C. et al. Temperature dependence of pure CsI: scintillation light yield and decay time. Nucl. Instrum. Methods A 480, 494-500 (2002).

16. Charlton, M. \& Humberston, J. W. Positron Physics (Cambridge Univ. Press, Cambridge, 2001).

17. Gabrielse, G. et al. First positron cooling of antiprotons. Phys. Lett. B 507, 1-6 (2001).

18. Calligarich, E., Dolfini, R., Genoni, M. \& Rotondi, A. A fast algorithm for vertex estimation. Nucl. Instrum. Methods A 311, 151-155 (1992).

\section{Acknowledgements}

The authors comprise the ATHENA Collaboration. We would like to thank G. Bendiscioli, S. Bricola, P. Chiggiato, J. Hansen, H. Higaki, A. Lanza, C. Marciano, O. Meshkov, P. Salvini, G. Sobrero, B. Schmid and E. Søndergaard. We also thank the CERN AD team and C. Surko, who provided essential advice. This work was supported by Istituto

Nazionale di Fisica Nucleare (Italy), Conselho Nacional de Desenvolvimento Científico e Technológico, Fundação de Amparo a Pesquisa do Estado do Rio de Janeiro (FAPERJ) e Fundação CCMN/UFRJ (Brazil), Grant-in-Aid for Creative Basic Research of

Monbukagakusho (Japan), the Swiss National Science Foundation, the Danish Natural Science Research Council, The UK Engineering and Physical Sciences Research Council (EPSRC), The EU (Eurotraps Network), and the Royal Society. L.V.J., M.H.H., M.C. and J.S.H. acknowledge the work of the late B. Deutch.

\section{Competing interests statement}

The authors declare that they have no competing financial interests.

Correspondence and requests for material should be addressed to J.S.H. (e-mail: jeffrey.hangst@cern.ch). 